\title{
Management of breast cancer patients leptomeningeal carcinomatosis: experience with Ommaya catheter
}

Felipe de Lacerda Pereira 1", Letícia Assad Maia Sandoval 2, Luiza Bernardes Ferreira 3, Ana Paula Teixeira Leite 3, Juliana Moreira Batista 3, Tatiana Strava Corrêa 4

${ }^{1}$ Medical School, University Center of Brasília, Federal District, DF, Brazil.

${ }^{2}$ Medical School, University of Brasília, Federal District, DF, Brazil.

${ }^{3}$ Medical School, Catholic University of Brasília, Federal District, DF, Brazil.

${ }^{4}$ Medical Oncology, Sírio-Libanês Hospital, Federal District, DF, Brazil.

${ }^{*}$ Corresponding author: Felipe de Lacerda Pereira. Octogonal 4, Bl F, 116. Zip Code: 70660-046 Federal District, DF, Brazil. Phone: +55 (61) 9 9947-7400. E-mail: felipelacerdape@gmail.com.

Research Ethics Committee Approval (if necessary): We declare that the patient approved the study by signing an informed consent form and the study followed the ethical guidelines established by the Declaration of Helsinki.

Received on: Jul 25, 2021. Accepted on: Aug 10, 2021. Available online: Aug 11, 2021.

\section{Abstract}

Leptomeningeal Carcinomatosis is a recurrent complication in different types of tumors with systemic involvement, especially breast cancers. Thus, given the different forms of treatment for this disease, this article presents the effectiveness of using Ommaya catheter as a way of administering chemotherapy. Two cases of breast cancer were studied and their therapeutic evolution reported. Despite the poor prognosis associated with meningeal carcinomatosis, these cases had a disease response and control for a longer period than the expected median.

Keywords: Leptomeningeal Carcinomatosis; Chemotherapy; Ommaya Catheter.

\section{Introduction}

Leptomeningeal Carcinomatosis (LC) is a clinical entity acquired through tumor invasion of the leptomeningeal vasculature over the subarachnoid space, compromising the entire neural axis and generating multifocal neurological signs and symptoms [1, 2].
It occurs in $5 \%$ to $10 \%$ of patients with systemic tumors [3]. The primary tumor cells can range from leukemias, lymphomas or solid tumors $[2,4]$. The diagnostic is made by locating the meningeal metastasis in the magnetic resonance imaging (MRI) and cerebrospinal fluid (CSF) cytology analysis. Assessment of the extent of 
disease of the CNS is important because large-volume subarachnoid disease or CSF flow obstruction is prognostically significant [3].

Traditional treatments options are radiotherapy and intra-CSF fluid therapy (either intraventricular or intralumbar) or systemic therapy $[5,6]$. More than $70 \%$ of patients with neoplastic meningitis have progressive systemic disease and consequently treatment is palliative and tumor response is of restricted durability The Ommaya reservoir should be preferred over lumbar puncture for intra CFS treatment, if it is an available resource [5].

There are many advantages of using this technique compared to intrathecal administration, such as providing greater patient comfort, as, usually, chemotherapy requires more than one session [6]. However, complications in the placement of the Ommaya reservoir as infections, intracranial hemorrhage are a limiting step of this technique. If performed by an experienced neurosurgeon, catheter implantation has very low complication rates. Therefore, intraoperative confirmation of the position of the catheter with fluoroscopic and computed tomography in the postoperative period prior is necessary. These 2 cases reports discuss the treatment of meningeal carcinomatosis in the scenario of patients with advanced breast cancer.

\section{Case report}

A 39-year-old woman patient, attends in first consult with history of metastatic lobular breast cancer (LBC) to skin, lymph nodes, bones, lung, liver. The immunohistochemical results of the patient's first biopsy demonstrated estrogen and progesterone receptors positive, HER2 negative, Ki67 40\%. The patient was previously treated with subsequent progression to several chemotherapies (CT) and hormone therapies (doxorubicin, paclitaxel, capecitabine, carboplatin, gencitabin, everolimus and exemestane, palbociclib and fulvestrant).

The patient complains of dizziness, headaches, and weakness of lower limbs. The diagnostic of meningeal carcinomatosis was confirmed by CSF analyses with neoplastic cells. She started intra CSF CT (methotrexate weekly) by an Ommaya catheter and systemic eribulin. The toxicity with IT-MTX was mainly nauseas, asthenia, and neutropenia, which were manageable with antiemetic and dose adjustment. She did not need support of granulocyte growth factors. Initially she had great improvement of neurologic symptoms, mainly of the headaches, but after 5 months the neoplastic cell score on CSF increased 
and the patient got performance worsening.

WBRT (whole brain radiotherapy) was initiated but patient did not complete because of worsening headaches. New PET scan shows disease progression even in new locals as pleura and bladder. At this time, we started best supportive care and she died after 2 months.

We also report a second case of Ommaya catheter used in leptomeningeal cancer. This second patient was female, 46 years, reported nodule in right breast and right axillary lymph nodes. The patient had a biopsy that shows triple negative breast cancer (TNBC), Ki67 90\%. Initial PET scan shows disease in breast and lymph nodes, no distant metastasis. She had neoadjuvant treatment with 4 cycles of dose dense doxorubicin and cyclophosphamide (AC), followed by 12 weeks of paclitaxel.

New PET scan with radiologic complete response and no other abnormalities. Before the scheduled surgery she complains of headache. Skull MRI shows meningeal thickening. The CSF exhibits neoplastic cells. As soon as she performed an Ommaya catheter implant, intra CSF chemotherapy with methotrexate (ITMTX) was started with methotrexate weekly and systemic oral capecitabine. There was a clinical benefit as well as reduction of the neoplastic cells score at CSF after 3 applications of MTX. The toxicity with IT-MTX was mainly nauseas and vomiting, which were treated with dexamethasone and antiemetics.

Cranial radiotherapy was considered at that time, but patient refused because of possible memory side effects. Patient completed 7 months of IT-MTX, with no evidence of disease elsewhere than meningeal. There was an increase in cells score at CSF and MRI confirms progression on several meningeal metastasis. Clinically, patients complained of headache and double vision. There was still no evidence of other distant metastasis. She started WBRT and systemic chemotherapy was modified to nabpaclitaxel plus atezolizumab, as the breast biopsy was positive PD-L1. Unfortunately, she evolved with worsening headache, without clinical improvements after radiotherapy. Then, best supportive care was indicated and, unfortunately, she died within 3 weeks.

\section{Discussion and Conclusion}

Two cases were evaluated with diagnosis of LC in different moments of the disease. One classic case with long period of systemic treatment for hormonal positive lobular breast cancer and another case of TNBC after neoadjuvant treatment with no further 
metastasis. Both had clinical benefit of treatment with Ommaya reservoir [7]. In our center, the standard treatment of LC is WBRT with or without intrathecal chemotherapy (IT-CT). The usual IT-CT regimen is methotrexate [1]. The expected adverse events associated with this therapy are aseptic meningitis, headaches, neck rigid, back pain, nausea, vomiting, fever, and lethargy. Less frequently, there may be transverse myelopathy, leukoencephalopathy and encephalopathy [8].

It was given $10 \mathrm{mg} / \mathrm{m} 2$ twice weekly until the cerebrospinal fluid cytology became negative and then gradually decreased, first to a weekly course, and if possible monthly if cytology remains negative. The IT-CT is applied by the clinical oncologist or the neurosurgeon in our center. To reduce the risk of worsening headaches and vomiting after IT-CT with MTX, it is recommended to prescribe intravenous or oral antiemetics before the CT and dilute dexamethasone $2 \mathrm{mg}$ with the MTX that will be applied in the Ommaya catheter. These two patients experienced no complications related to catheter implantation.

The first case actually experienced improvement of headaches soon after the Ommaya implantation, even without the IT-MTX, because it was possible to drain the CSF and reduce the intracranial pressure. Both patients had clinical benefit with ITMTX and manageable toxicities. Prognosis of LC remains poor, with median survival of 4 months for patients receiving classic treatments [9]. The main factors associated with survival are performance status at diagnosis, CSF protein level and TNBC subtype. The current management of LC consists of a combination of intra-CSF CT, systemic therapy, radiotherapy and bestsupportive care (Figure 1).

As exposed, there are many advantages of using Ommaya reservoir compared to lumbar puncture for intra CSF CT, such as greater patient comfort, preventing drug leakage and improve its distribution. Nevertheless, the treatment of meningeal carcinomatosis remains a challenge for clinicians. Therefore, we support new clinical trials for this serious evolution of breast cancer. 
Figure 1: Leptomeningeal carcinomatosis diagnosis and treatment.

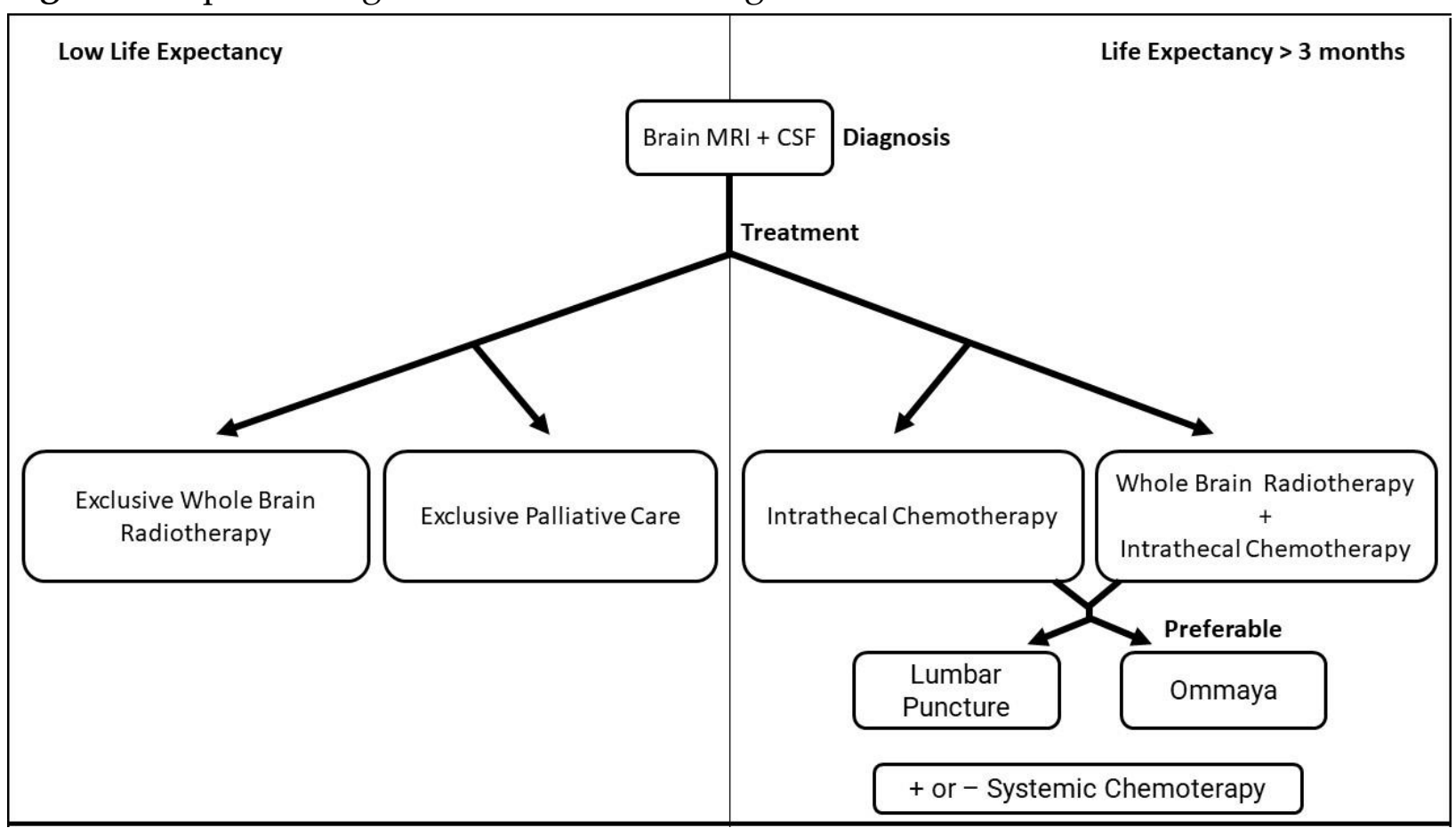

Legend: MRI (magnetic resonance imaging); CSF (cerebrospinal fluid).

\section{References}

[1] Katz A, Marques R, Novis Y, Rocha V, Costa FP, Fernandes GS, Ferrari CLS, Hoff PMG. Oncologia Clínica: Terapia Baseada em Evidências [2.ed.]. SÃO PAULO: SBSHSL - Sociedade Beneficiente de Senhoras Hospital SírioLibanês, 2014:624.

[2] Martins SJ, Azevedo CRAS, Chinen LTD, Peterlevitz MA, Gimenes DL. Meningeal Carcinomatosis in Solid Tumors. 2011;69(6):973-80. [3] Gleissner B, Chamberlain MC. Neoplastic meningitis. Lancet Neurol. 2006 May;5(5):443-52. doi: 10.1016/S14744422(06)70443-4.

[4] Grossman SA, Krabak MJ. Leptomeningeal carcinomatosis. Cancer
Treat Rev. 1999 Apr;25(2):103-19. doi: 10.1053/ctrv.1999.0119.

[5] Montes de Oca Delgado M, Cacho Díaz B, Santos Zambrano J, Guerrero Juárez V, López Martínez MS, Castro Martínez E, Avendaño Méndez-Padilla J, Mejía Pérez S, Reyes Moreno I, Gutiérrez Aceves A, González Aguilar A. The Comparative Treatment of Intraventricular Chemotherapy by Ommaya Reservoir vs. Lumbar Puncture in Patients With Leptomeningeal Carcinomatosis. Front Oncol. 2018 Nov 20;8:509. doi: 10.3389/fonc.2018.00509.

[6] Gwak HS, Lee SH, Park WS, Shin $\mathrm{SH}$, Yoo $\mathrm{H}$, Lee SH. Recent Advancements of Treatment for Leptomeningeal Carcinomatosis. J 
Korean Neurosurg Soc. 2015 Jul;58(1):18. doi: 10.3340/jkns.2015.58.1.1.

[7] Sandberg DI, Bilsky MH, Souweidane MM, Bzdil J, Gutin PH. Ommaya reservoirs for the treatment of leptomeningeal metastases. Neurosurgery. 2000 Jul;47(1):49-54; discussion 54-5. doi: 10.1097/00006123200007000-00011.

[8] Wen PY, Plotkin SR. Neurologic complications of cancer chemotherapy. UpToDate, 2006. Available at: www.uptodate.com. Accessed 27 April 2006.https://www.uptodate.com/content s/overview-of-neurologic-complicationsof-conventional-non-platinum-cancerchemotherapy

[9] Kim H, Lee EM. A Retrospective Analysis of the Clinical Outcomes of Leptomeningeal Metastasis in Patients with Solid Tumors. Brain Tumor Res Treat. 2018 Oct;6(2):54-59. doi: 10.14791/btrt.2018.6.e12.

Conflict of interest: Declare that there is no conflict of interest.

Acknowledgements: to all the health care worker involved in the supportive care of these patients.

Funding: None.

How to cite this article: Pereira FL, Sandoval LAM, Ferreira LB, Leite APT, Batista JM, Corrêa TS. Management of breast cancer patients leptomeningeal carcinomatosis: experience with
Ommaya catheter. Brazilian Journal of Case Reports. 2021Jul-Sep;01(3):125-130. 